This is a post-peer-review, pre-copyedit version of an article published in Journal of Intelligent \& Robotic Systems. The final authenticated version is available online at: https://doi.org/10.1007/ s10846-017-0750-z

\title{
Development of a new hydraulic ankle for HYDRÖ̈D humanoid robot
}

\author{
A.Abdellatif · S.Alfayad · Arne-Christoph \\ Hildebrandt * F.B. Ouezdou · N.Mechbal . \\ Yahya Zweiri
}

Received: date / Accepted: date

\begin{abstract}
For humanoid robots, design of the ankle mechanism is still open research problem since high torque is required while compact structures have to be maintained. This paper investigates an enhanced design of 3 degree-of-freedom hydraulic hybrid ankle mechanism. The design is based on (US9327785) [1]. Using a hybrid kinematic structure with hydraulic actuation, allows us to reach a slender humanoid ankle shape while enabling the high torque performances required for stable walking. Performances analysis of the first version ankle mechanism designed for HYDROï humanoid robot showed some limits mainly induced by seal friction and pistons misalignment. In this paper, the influence of the friction parameters is explored. A virtual model is developed to evaluate the performances of a new flexion/extension and adduction/abduction pistons arrangement. Then, a control algorithm is simulated and implemented, as an example, to the flexion/extension motion of the new ankle mechanism. Finally, an experimental validation for the performances of the new proposed hydraulic ankle is conducted using the built hardware prototype, the results show significant improvement.
\end{abstract}

Keywords Hydraulic actuation · Humanoid Robots · HYDROiD Robot · Friction Analysis $\cdot$ Experimental Validation

\section{Introduction}

Nowadays, the need for intelligent and robust humanoid robots has become crucial, especially in the areas of research platforms, industrial services, investigation of hostile environments and human-robot interaction [2]. Humanoid robots have recently

\footnotetext{
S.Alfayad

10/12 avenue de l'Europe, 78140 Vélizy, France

Tel.: +33(0)139254970

Fax: +33(0)139254985

E-mail: samer.alfayad@lisv.uvsq.fr
} 
attracted more research attention in the field of walking on rough terrain and in unstructured environment as well as studying human locomotion behavior [3]. Moreover, acceptance may increase when familiar human-like robots are used for performing tasks in domestic environment and for entertainment purpose. However, there are some challenges to consider in the humanoid design such as reducing energy consumption that may limit robot performances, safe human-robot interaction as well as physical compliance in the robot behavior. In order to tackle these issues, increasing research is focused on the humanoid robot actuation technologies. Two main actuation technologies are used for humanoid robots.

Firstly, electric actuation, used in several humanoid robots such as HONDA ASIMO [4], NAO [5], LOLA [6] and WABIAN-2 [7]. Electric actuators are normally lowcost. However, they need a gearbox to adapt the required torque and speed. This induces many of intermediate mechanical parts. Therefore, this increases substantially the total weight of the system. Indeed, actions like jumping and running need a high dynamic actuation performance in terms of speed and torque which can not always be supported by electric motors unless to oversize them.

The other technology used for actuating humanoid robots is based on hydraulics. The advantages of this technology lies in its very high power-to-mass-ratio, in addition to its high power-to-volume-index compared to the electric actuators. This brings efficient solution in term of high speed, robustness and the ability to cope with high transient force which are required for humanoid robots [8]. This technology was adopted to actuate robots like: CB [9], SARCOS [10], HyQ[11] and ATLAS [12] humanoid robots. The robustness and high performances shown by these robots validated that hydraulic actuation is suitable for legged robots.

Nevertheless, hydraulic actuation has some drawbacks. Hydraulic actuation systems always suffer from internal leakage inside the actuators as well as possible losses through hoses and connectors. Moreover, a central Hydraulic Power Unit (HPU) that produces high pressure is usually required for heavy duty applications. HPU based solution is more energy efficient than other solutions since a locked joint at a given position leads inherently to bypass the corresponding hydraulic actuator. In, this case, the energy produced by the HPU is converted to heat dissipation in hydraulic fluid. This may change the fluid physical and chemical properties making it hard to adjust the parameters of the control algorithms. To tackle this major drawback of central hydraulic actuation, an Integrated Electro-Hydraulic Actuator (IEHA) was developed by Alfayad et al. [13] in which it is possible to actuate each joint of a robot by its own hydraulic unit and independently from the other joints. Moreover, the Concept of Integrated Hydraulic Connections (CIHC) is developed for HYDROiD humanoid robot. The CIHC consists passes fluid inside the links parts, thus, eliminating the need for external hoses and reducing their possible leakage.

Furthermore, one of the main mechanisms required to achieve legged locomotion is the ankle joint. Knowing that bipedal humanoid robots are expected to play a major role in the future, several researchers have conducted a study for ankle mechanisms 
of humanoid robots. Ogata et al. [14] have studied the fundamental characteristics for an effective ankle mechanism, which includes: light weight and robustness, the ability of adaption to the ground, torque generation after landing of the foot and the sensing elements. Syrseloudis et al. [15] investigated several robotic designs for ankle function evaluation, measurement and physiotherapy. They suggested parallel tripod mechanism with an extra rotation axis based on kinematic analysis and ensured the simplicity, accuracy and generality of their solution experimentally.

Aggogeri et al. [16] introduced an ankle kinematic model and used a simulation software to demonstrate the ankle movements in his attempt to produce a rehabilitation device for the human ankle based on a parallel mechanism. Another example for the design of the ankle joint using a parallel mechanism is introduced in the humanoid robot TaeMu [17]. In this robot, the sagittal and the frontal rotations of the ankle are achieved using two coupled hydraulic actuators. In this design, for every given joint angle, velocity and torque, one can compute the cylinder linear displacement and velocity and vice versa. Another novel design for the ankle was introduced by Omer et al. [18] in which an adjustable stiffness artificial tendon was introduced for the ankle joint of a bipedal robot. Narioka et al. [19] proposed a robotic ankle-foot complex, which is similar to the anatomy of the human foot. The proposed foot consisted of three links, two joints and four plantar muscles, whose mechanical stiffness can be controlled by utilizing McKibben pneumatic actuators. Eventually, this mechanism performed the biped motion successfully.

On the other hand, Ouezdou et al. [20] analyzed different foot models using dynamic simulation to study their performance in walking gait. The two studied criteria were the energy consumption and the normal contact forces. Their results proved the efficiency of the active flexible foot model and that the introduction of an active joint at the toe level reduces the required energy for walking gait. Further research on the ankle mechanism design was carried out by David et al. [21], in which they proposed a compliant soles which can absorb the perturbations generated by ground impact forces. This mechanism was applied to the humanoid robot HRP-2.

Moreover, Yousheng et al. [22] showed the effects of geometry and design parameters of the hydraulic leg mechanism on the performance of the hydraulic actuation system. Improvements in the mechanical design always have a great influence on the power required for the actuation system as well as on the enhancement of the whole system performances.

Furthermore, E. M. Ficanha et al. [23] suggested the usage of cables to control two degrees of freedom of the ankle, in which power, range of motion and light weight can be provided. The developed mechanism showed a passive mechanical impedance close to the impedance of a human ankle, prompting the feasibility of controlling this mechanism using impedance controller.

Based on the state of the art of ankle mechanism design and as reported by the authors [24], the design of the ankle mechanism raises usually two global questions 
to answer: What is the real number of degrees of freedom to reproduce? Then, which actuation technology will be used? The solution proposed for HYDROiD humanoid robot ankle is based on a spherical 3 DoFs mechanism actuated hydraulically. The adopted solution avoids the "blow up" issue of the ankle inherently induced by the use of gear-box reduction system of the major humanoid robots. Moreover, the slender shape for the HYDROiD ankle induces an advantage regards the energy consumption since its center of mass is close to knee joint.

In this paper, a brief description of the hybrid ankle mechanism for HYDROiD is discussed in section 2. Experimental prototype and characterization data of this version are detailed in section 2.2. Moreover, the basic phenomena that limit performance in hydraulic robotics such as leakage, seal friction and piston misalignment are identified. Based on a developed virtual model detailed in section 3, one specific focus dedicated to the study of the friction parameters affecting the ankle joint motion is given in section 3.3. Based on the analysis of the previous data, a new improved solution of the ankle which can be fit in compact space is presented in section 4. A control algorithm is simulated then experimentally implmented, as an example, to the flexion/extension motion of the ankle is presented in section 5. This leads to the experimental validation of the performances of the new version. Finally, section 7 is devoted to the conclusion and the future work.

\section{Hybrid ankle mechanism description and performance analysis}

\subsection{Mechanism description}

Hybrid mechanisms is one of the main advantages of the humanoid robot HYDROiD [25]. In this paper, the hybrid ankle mechanism is introduced. This mechanism consists of a mechanical system with an external hydraulic circuit, the mechanical system composes of two subsystems. The first subsystem is a serial chain mechanism, while the second subsystem is a fully parallel mechanism. The latter can be described as a series of rigid bodies connected to two platforms (i.e. fixed and moving), as stated by Gough-Stewart [26].

The simplified ankle mechanism sketch shown in Fig.1, consists of a top yaw rotation joint denoted by $\theta_{z}$, while the pitch and the roll rotations are in the bottom and denoted by $\theta_{x}$ and $\theta_{y}$ respectively. A fourth rotation $\theta_{\text {toe }}$ allows HYDROiD to have an active foot as suggested by Ouezdou et al. [20]. To actuate the full parallel subsystem, four linear actuators $r^{j}$, performing the coupled motions with the connected cables denoted as $C_{i}$ with $i \in 1,2,3,4$ are added. One has to notice that, for this first design, generating either flexion/extension or adduction/abduction pure motions, only two antagonistic linear actuators have to be used to pull the steel wired cables fixed to the foot. Moreover, the hydraulic system consists of four single acting cylinders that are controlled by two double stage servo-valves. As shown in Fig.2, each two coupled cylinders work together to actuate the foot in the sagittal or the frontal planes. The 
front and back hydraulic pistons, denoted as $r_{1}$ and $r_{3}$, are hydraulically connected to one servo valve therefore it behaves like one double-acting cylinder. As the fluid enters the stator from below, the piston moves upwards, releasing the air through outlets located on the top of the stator. The corresponding piston is connected to the return line which causes downwards motion making the whole foot moves freely around the universal joint. The same principle is applied to the left piston $r_{2}$ and right one $r_{4}$ giving the rotation in the frontal plane. Moreover, aligning the four pistons axes along the tibia vertical axis allows us to fit the hydraulic subsystem in a very compact space and ensure a slender ankle shape.

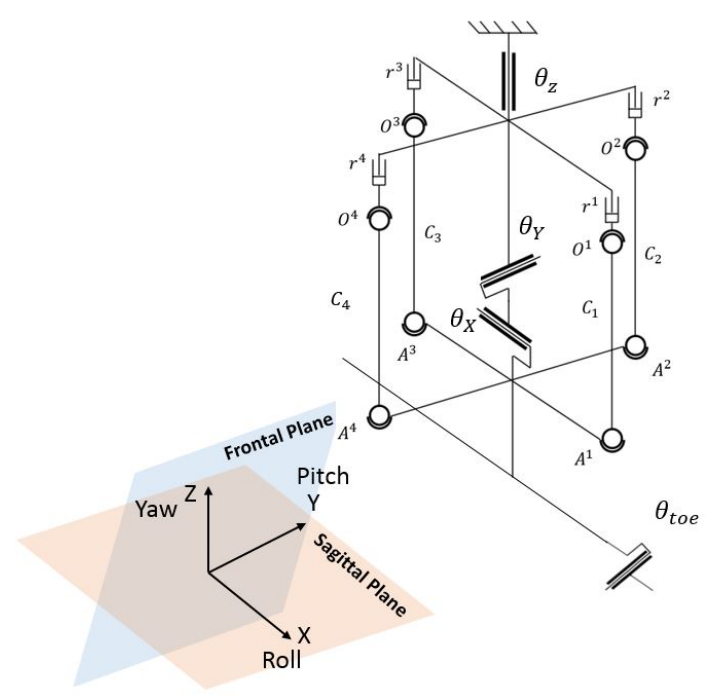

Fig. 1 Simplified kinematic structure of hybrid ankle mechanism

Finally, the double-acting cylinder $L_{5}$ shown on Fig. 2 is dedicated to the yaw motion of the whole system formed by the tibia and the active foot relative to the knee. The first ankle prototype is shown in Fig.3.

\subsection{Performance Analysis}

As reported in [24], a prototype was built and tested. The target of the developed mechanism is to reach the following ranges of motion. The rotation $\theta_{z}$, around the vertical axis is between $-45^{\circ}$ and $+45^{\circ}$ while $\theta_{x}$ in the frontal plane is between $-20^{\circ}$ and $+20^{\circ}$ and $\theta_{y}$ in the sagittal plane is between $-15^{\circ}$ and $+45^{\circ}$. At first, an analysis is carried out to identify the parameters that may affect the hydraulic performance of the hybrid ankle mechanism. The identified parameters with significant influence are mainly the pressure to generate the required torque around the sagittal and frontal 


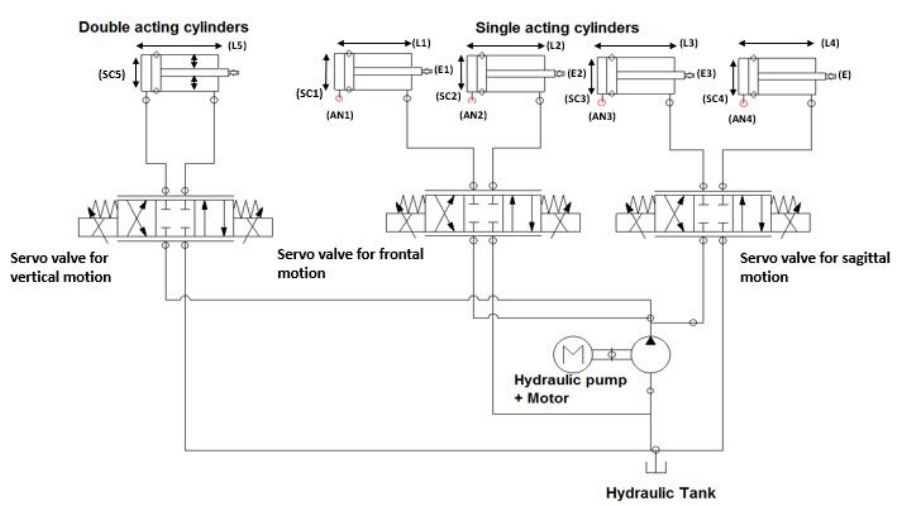

Fig. 2 Hydraulic subsystem diagram for the hybrid ankle in which piston stroke $(L(i))$, cylinder outer diameter $S C(i)$ with $(i=1,2,3,4,5)$, piston-cables connecting point $E(j)$ and air pressure outlet point $A N(j)$ with $(j=1,2,3,4)$

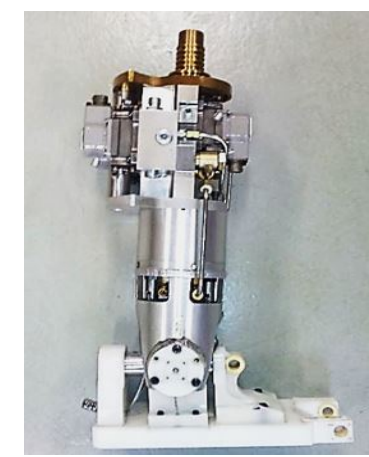

Fig. 3 First Ankle mechanism for the hydraulic humanoid robot HYDROïD

axes, the leakage at the piston level and the friction of the hydraulic pistons. The increase of friction values leads inherently to wear and more energy lost. The wearing phenomenon was clearly identified through the first version of the hybrid ankle mechanism.

Concerning the required pressure, it is observed that high pressure values were necessary to satisfy the required motions and forces. Hence, various mechanical parts need to be redesigned. Decreasing operating pressure will be useful to reduce the size of the required HPU and hence reducing the total weight of the humanoid robot and increase the mobility of the latter.

For the leakage at the piston level, O-ring are usually placed on pistons to prevent oil leakage. Under hydraulic pressure, O-ring will undergo elastic deformation increasing friction with the cylinder chamber and finally preventing leakage. Reducing the friction between the O-ring and the cylinder chamber while ensuring zero leakage is a trade-off which is hard to achieve. Therefore, special attention needs to be paid to 
the mechanical design of the piston and the cylinder. Reducing the theoretical leakage (without the O-ring), is a key factor to ensure almost zero leakage with minimum friction when the O-ring is used. Therefore, the theoretical leakage flow $Q_{\text {leakage }}$ has to be calculated depending on the designed parts. The leakage mainly depends on the shape of the moving actuators namely the hydraulic pistons moving in the cylinders. Thus, based on the shape of the piston, hydraulic fluid physical properties and pressure difference between the two cylinder chambers, the resultant leakage flow without considering the effect of the O-ring can be calculated as follows, Rabie[27]:

$$
Q_{\text {leakage }}=\frac{\Delta P \times \pi \times d \times c^{3}}{12 \times \rho \times v \times L} \text {. }
$$

Where $d$ is the piston diameter in $[m], c$ is the radial clearance in $[m], \rho$ is the density of the hydraulic fluid in $\left[\mathrm{kg} / \mathrm{m}^{3}\right], v$ is the kinematic viscosity in $\left[\mathrm{m}^{2} / \mathrm{s}\right]$ and $L$ is the length of the piston in $[m]$. Using the corresponding values of the parameters of the first version of the ankle mechanism, the leakage flow is estimated to be equal to $0.02[l / \min ]$.

The third aspect pointed by the performance analysis of the first ankle mechanism is related to the misalignment effects of the hydraulic pistons. One of the most important parameters that always needs to be considered in moving mechanisms is friction. As some energy is lost due to friction in the form of heat, deformation or wear, this leads to a total degradation of system efficiency and lifetime with increasing costs as well. From the experimental analysis for the first version of the ankle mechanism, it was observed that the motion of the cables exerts a radial force that acts on the piston inside the cylinder. This force creates a misalignment of the piston as shown in Fig.4 with respect to the cylinder axis by an angle $\alpha$ [28]. This angle increases piston friction, wear of the seal and oil leakage.

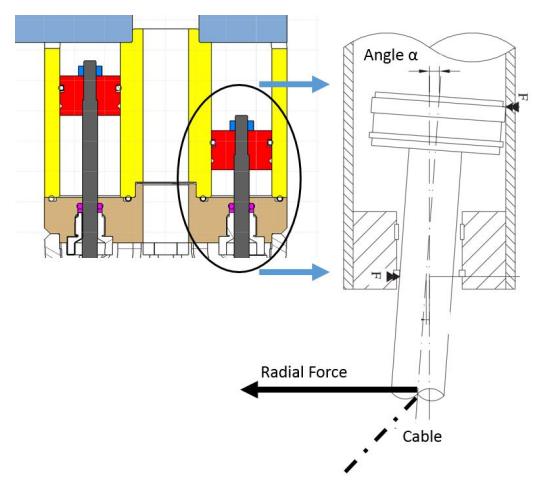

Fig. 4 The misalignment caused by the force resulting from cables action 
2.3 Summary of performance analysis for the ankle mechanism

Based on all of the above discussed parameters, a high relative pressure is needed to actuate this mechanism since the first version of the ankle mechanism suffers from relatively high oil leakage and friction. Hence, it's necessary to design and manufacture a modified version of the mechanism to enhance these parameters. To achieve this aim, a specific focus is dedicated to the friction phenomenon. In order to estimate the friction effects, a dynamic simulation has to be carried out. This simulation can be conducted either using commercial software (i.e. ADAMS) or developing our own model. Using ADAMS software has an advantage since the friction is based on the latest improved model where some parameters have to be tuned in "trial and error" procedure. Hence, a complete virtual model of the ankle mechanism based on the ADAMS software was adopted and the whole procedure is detailed in the next section.

\section{Virtual Model and Friction Analysis of the Ankle Mechanism}

\subsection{Virtual model}

To fulfill the requirement of efficiency of a virtual model, it's necessary to develop a the geometrical model for the mechanism using a CAD (Computer Aided Design) software. In this study, the geometric model was developed which represents the basis for the construction of the real mechanism. Then the developed model is interfaced with ADAMS as shown in Fig5.

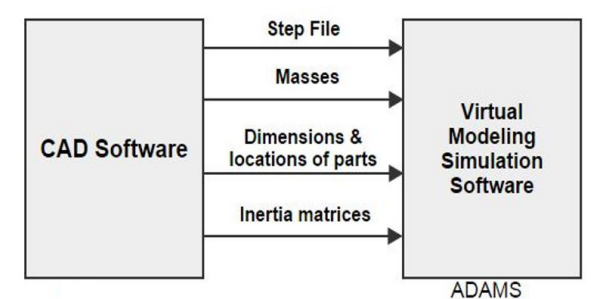

Fig. 5 Interaction between CAD and ADAMS for building and analyzing performances of proposed solution

To deal with hydraulic parameters, ADAMS offers a toolbox called EASY5 which represents the mathematical modeling of a real hydraulic system composed with a hydraulic servo valve, hydraulic oil density, and pump nominal pressure. This allows ADAMS to emulate the real systems, where its hydraulic parameters can be set numerically. Fig.6 shows a screenshot of the hydraulic circuit drawn in the toolbox and used to simulate the new hybrid ankle mechanism. 


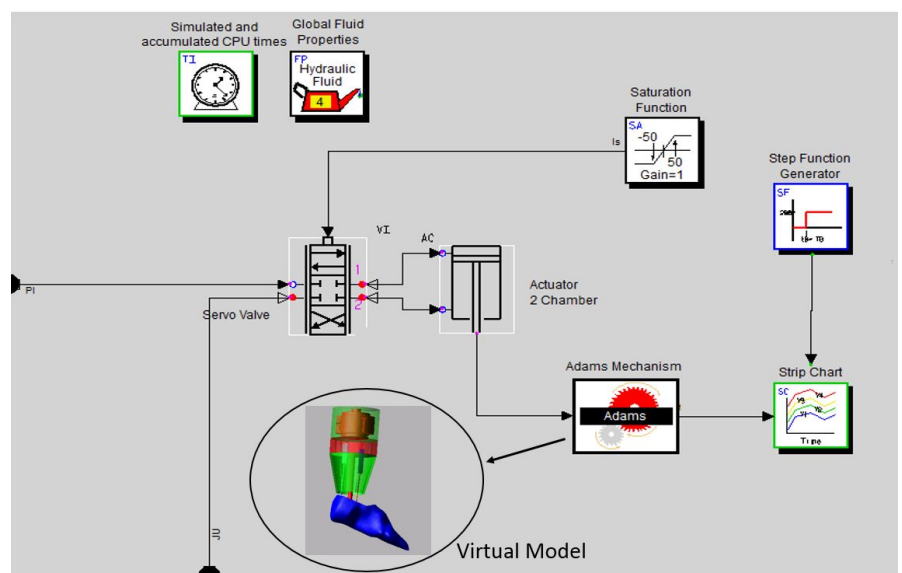

Fig. 6 Easy5 toolbox for the mathematical modeling of new hybrid hydraulic ankle mechanism with the virtual model based on ADAMS software where each cable is modeled by a rigid body and two spherical joints.

The system model components are the servo valves having a pressure drop $\Delta P=$ 50 [bar $]$, with nominal flow rate equals to $12[\mathrm{l} / \mathrm{min}]$. Furthermore, the pump is modeled as a constant pressure source, while the hydraulic pistons are assumed having a pressure area of $A_{p}=294.5\left[\mathrm{~mm}^{2}\right]$. The used hydraulic oil has a bulk modulus $\beta=1.5[\mathrm{GPa}]$ and density $\rho=850\left[\mathrm{~kg} / \mathrm{m}^{2}\right]$. Since the frequency for the periodic walking HYDROiD robot is about $1[\mathrm{~Hz}]$ [24], no high frequency actuation of the servo valve is needed. Therefore, the transfer function for the servo valve can be expressed as a first order equation ([29] and [30]). Hence, the system transfer function can be expressed as following:

$$
G(s)=\frac{x_{v}(s)}{I(s)}=\frac{K}{T s+1}=\frac{3.7268 e-08}{0.0015 s+1}
$$

Where $x_{v}(\mathrm{~s})$ and $I(\mathrm{~s})$ are the laplace transformation of the spool displacement of servo valve and the servo-valve input current respectively. $K$ is the static flow gain and $T$ is the time constant.

\subsection{Position Control}

A PID control loop is applied on the virtual model (See Fig.7) to let the ankle mechanism following the desired motion. The inputs of this control loop are the desired angles $q_{d}=\left(\theta_{x}, \theta_{y}\right.$, and $\left.\theta_{z}\right)$ and the outputs are the measured ankle angles $q_{m}$.

In order to apply the control algorithm, the model in ADAMS is connected to a $\mathrm{C}++$ subroutine where the control law is written. The controller was firstly applied to the pitch rotation angle $\theta_{y}$ as an example. Fig. 8 shows the response of the system for a step amplitude equals to $0.5[\mathrm{rad}]$. It is observed that the system reached stabilized state with settling time equals approximately to $3.9[\mathrm{~ms}]$ and with zero steady state 


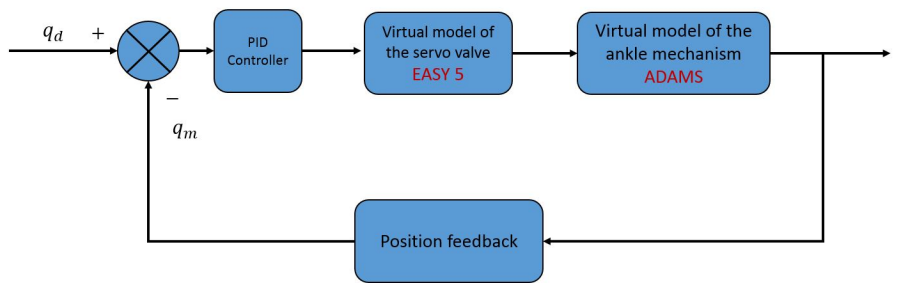

Fig. 7 Control loop of the ankle mechanism used for the ankle virtual model.

error.

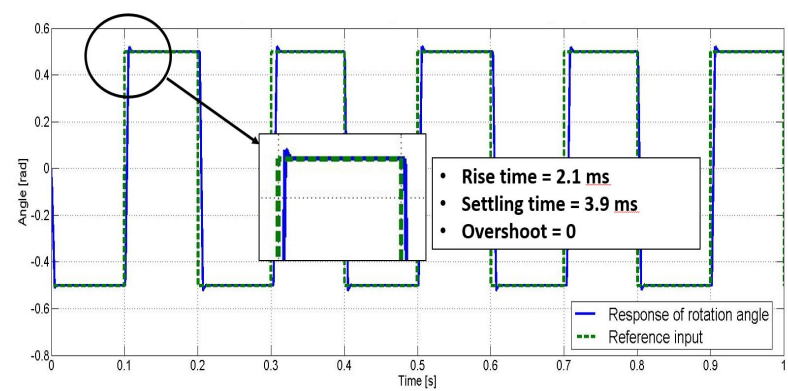

Fig. 8 Step response of $\theta_{y}$ for a step input of $0.5[\mathrm{rad}]$. The response signal settles after $3.9[\mathrm{~ms}]$

\subsection{Friction study of ankle mechanism}

Previous studies shows that the effects of friction are most noticeable at the pistons for hydraulic applications [31]. This leads to the integration of friction in the virtual model with the aim of making it more realistic. A friction model built in ADAMS, based on the study developed by Ellman et al. [32], is used to simulate the behavior of the ankle actuators. This model is concerned with the transition phase between the rest position of the actuator and the beginning of its motion. The transition phase is detailed on Fig.9.

The implemented model is governed by the following equation:

$$
F_{f_{i}}=\left(1-\mu_{f} \frac{\min \left(\left|v_{p_{i}}\right|, v_{f}\right)}{v_{f}}\right) F_{s}+c_{f} v_{p_{i}}
$$

Where $F_{f_{i}}$ is the friction force which always operates against the direction of piston movement, $\mu_{f}$ is the coefficient of static friction, $v_{p_{i}}$ is the velocity of the piston $i$, $v_{f}$ is the velocity of the piston in the transition phase, $F_{s}$ is the force of static friction and $c_{f}$ is the coefficient of viscous friction. 


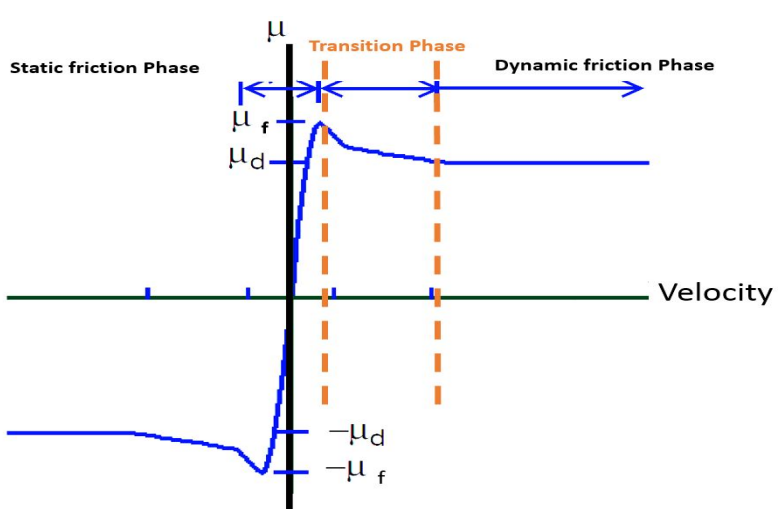

Fig. 9 The friction model response as stated by Ellman [32], in which the transition zone is clearly shown.

To get the friction parameters for Eq. 3, an experimental identification leads to a static friction force $F_{s}$ for the ankle first prototype equals to $52.5[N]$. Therefore, the values of $\mu_{f}$ and $c_{f}$ are chosen accordingly to $F_{s}$ and based on the material combination of the parts of the first ankle mechanism [31]. These values were found to be: $\mu_{f}=0.333$ and $c_{f}=0.0525[N . s / m m]$. On the other hand, the velocity of the piston in the transition $v_{f}$ phase was estimated $50[\mathrm{~mm} / \mathrm{s}]$.

Several simulations with different friction parameters were carried out in order to study their sensitivity to the static force $F_{S}$. The simulations showed clearly an important influence of the coefficient of the static friction $\mu_{f}$. No major influence of coefficients $c_{f}$ and $v_{f}$ were observed. The developed virtual model and the friction analysis results are used to propose new material combination for the enhanced version of the ankle, as detailed in the next section.

\section{New mechanism design}

In order to overcome the previous problems mentioned in section 2, (e.g pressure supply for producing required torques, friction force, leakage effects and misalignment of the pistons), a new version of the ankle mechanism is proposed. The new mechanism kinematics structure has to be modified while keeping the total number of degrees of freedom and the rotation around the vertical axis $\left(\theta_{z}\right)$ unchanged. The arrangement of two other degrees of freedom $\left(\theta_{x}\right.$ and $\left.\theta_{y}\right)$ has to be changed in order to increase the efficiency of the mechanism which was unsatisfactory. Moreover, avoiding the misalignment of the piston axes and simplifying the whole system are also targeted. In the next subsections the choices for the new proposed solution are presented. 
4.1 Modified kinematic structure

Since the main objective of the ankle mechanism is to achieve walking, therefore the walking gait cycle of human being was studied in [18]. This analysis concluded that a large torque is required for the flexion/extension motion of the ankle namely $\theta_{y}$ as depicted in Fig. 10 which assumes a human weighing $120[\mathrm{~kg}]$. This mass is targeted for the underdevelopment HYDROïD humanoid robot. Therefore the required maximal torque $\tau_{y}^{\max }$ is equal to $216[N . m]$. One should notice that the maximal required torque for the motion in the frontal plan $\tau_{x}^{\max }$ is around $101[N . m]$ which is less than $46 \%$ of $\tau_{y}^{\max }$.

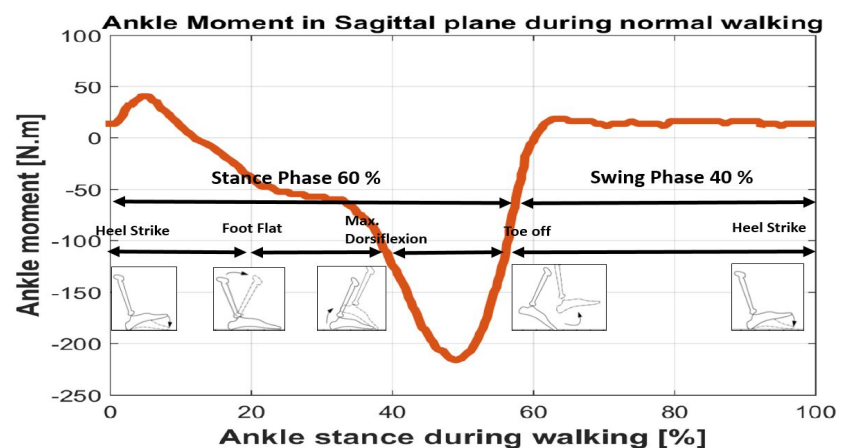

Fig. 10 The moment exerted by the human ankle during the sagittal rotation for a subject with a mass of $120 \mathrm{~kg}$

In order to optimize the produced torques by the ankle mechanism, it is necessary to make the two couples hydraulic pistons front\&back $\left(r_{1}, r_{3}\right)$ and left\&right $\left(r_{2}\right.$, $r_{4}$ ) contributing to produce the flexion/extension torque $\tau_{y}$. This will enhance the capabilities of the ankle mechanism but requires a complex control law to derive the coupling actions between the four hydraulic actuators. Rotating the two hydraulic pistons couples around the roll axis by an angle $\alpha$ leads to a new resulting torque $\tau_{y}^{\alpha}$ given by the following equation:

$$
\tau_{y}^{\alpha}=F_{f b} d \cos \alpha+F_{l r} d \sin \alpha
$$

Where $F_{f b}$ is the force produced by the front and back pistons while $F_{l r}$ is the force produced by the left and right ones. $d$ represents the distance between the hydraulic actuators and the tibia axes as depicted on Fig.11.

For a given HPU system, the available pressure is the same for all hydraulic actuators of the ankle mechanism. Therefore, assuming that the four pistons have the same section area, equation Eq. 4 can be re-written in the following form :

$$
\tau_{y}^{\alpha}=P S_{p} d(\cos \alpha+\sin \alpha)
$$




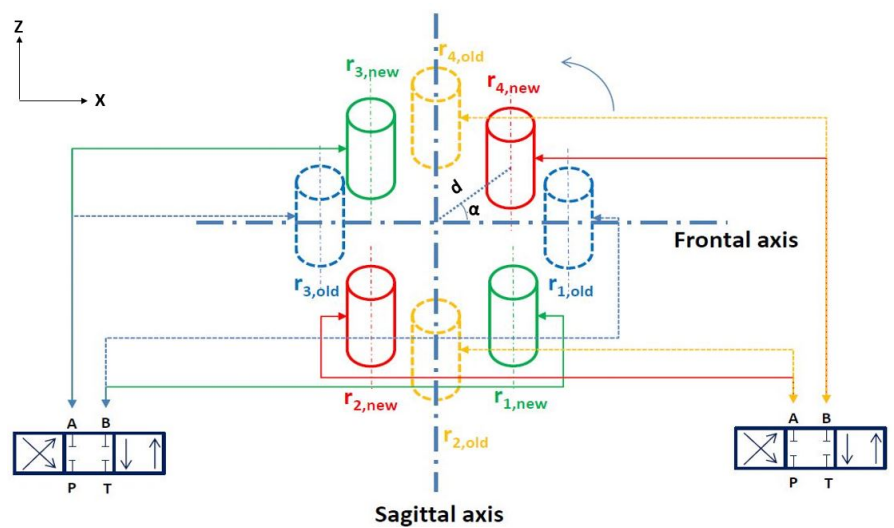

Fig. 11 The new position of the piston actuators rotated by angle $\alpha$ where the dotted lines describe the old position and the solid lines the new ones. The projection is on the X-Y plane where $\alpha$ is the angle between the axis of the actuating piston and the axis of the ankle and $d$ is the distance from center of the ankle to the center of the actuating piston

Where $P$ is the supplied pressure and $S_{p}$ is the section area of each hydraulic piston. It's clear that to get the maximum value of the flexion/extension torque for a given pressure, section area and $d$ distance, angle $\alpha$ should be equal to $\frac{\pi}{4}$ since this value maximize the sum $(\cos \alpha+\sin \alpha)$. Therefore, the new proposed parallel subsystem mechanism including the hydraulic actuators with their fixed cables rotated around the roll axis by an angle of $\frac{\pi}{4}$ as depicted on Fig. 12 .

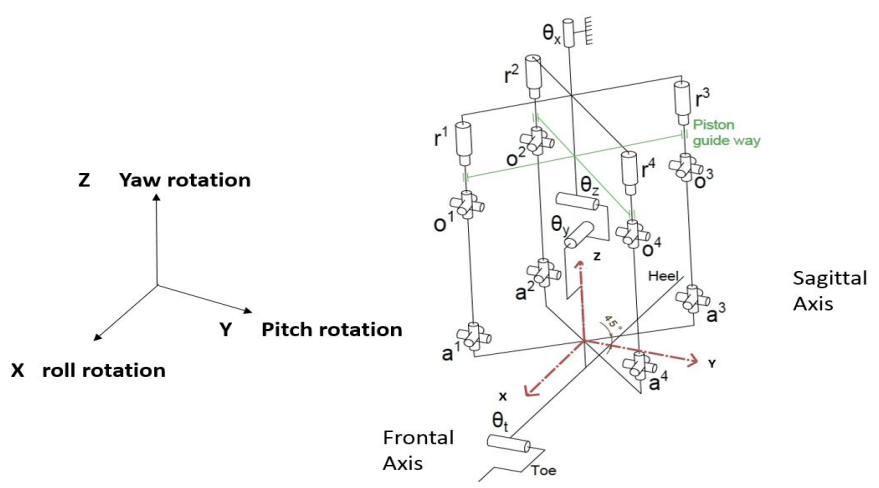

Fig. 12 The new kinematic structure of the enhanced ankle mechanism 
Table 1 Table showing material combinations for the enhanced ankle mechanism

\begin{tabular}{lllll}
\hline Key & Ankle Parts & Materials & $\mu_{f}$ & $c_{f}$ \\
\hline A & Tibia \& O-ring seal & Fortal \& PTFE & 0.24 & 0.19 \\
B & Hydraulic Piston rod \& Cover Nut & Steel \& Bronze & 0.18 & 0.16 \\
C & Hydraulic Piston rod \& Guide-way bearing & Steel \& PTFE & 0.2 & 0.05 \\
\hline
\end{tabular}

\subsection{Piston Friction Force Reduction}

Several aspects have to be considered for the selection of mechanical parts of the enhanced ankle mechanism. These aspects include the material selection, the used fluid, the surface finish, the operating temperature, and the surface coating (bushing). The most important choices have to consider the material combination of parts at the points of contact with the hydraulic pistons denoted $(A),(B)$ and $(C)$ which are shown on Fig.14. The new material combinations induced new friction parameters which are introduced in the virtual model (presented in section 3) to estimate the new friction forces on the pistons. Table 1 summarizes the corresponding parameters for the above mentioned three contact points. Different sinusoidal functions were tested and the new piston friction force minimal value was found to be $F_{\text {final }}=35[N]$ at contact point $(A)$. In Fig.13(a), the new forces of friction are shown for one of the front and one of the back pistons making the sagittal motion. While in Fig.13(b), this new obtained friction force was compared with other output simulation values (including the output friction force from the first prototype which is equal to 52.5 $\mathrm{N})$. These results validate the improvement of the new design in the term of friction reduction by almost $33 \%$. For point $(B)$, it was enhanced by $11 \%$ while for point $(C)$, an enhancement of $10 \%$ was achieved.

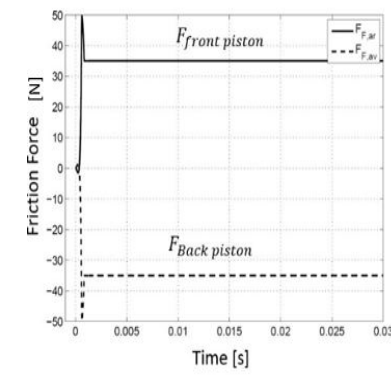

(a)

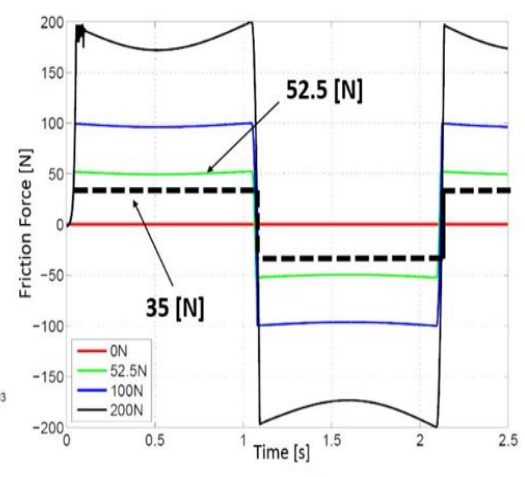

(b)

Fig. 13 Simulation results for the friction forces acting on the ankle pistons 


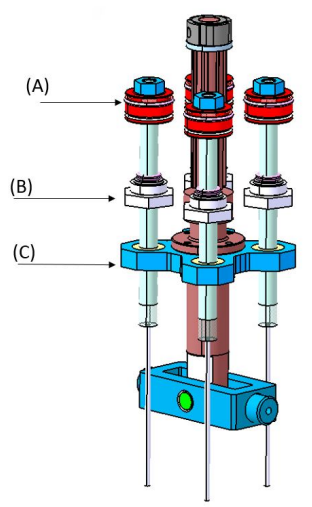

Fig. 14 Points of contact in the enhanced ankle mechanism defined in Table 1.

\subsection{Leakage and Misalignment issues}

The other tackled problem here is the oil leakage. Hence, an analysis of the O-ring effects on the performances of the actuators is carried out. The increase in the oil leakage is due to the mechanical design which makes the O-ring encounters an overwhelming leakage and so, more O-ring deformation is needed to overcome such leakage, causing more friction. Therefore, the leakage in the new design was reduced by enhancing the mechanical design of the piston, the rod and the cylinder. The piston radius has been increased from $2.5[\mathrm{~mm}]$ to $5[\mathrm{~mm}]$ and piston head radius from $10[\mathrm{~mm}]$ to $13[\mathrm{~mm}]$. This leads to a decrease in flow leakage to $15 \%$ of its previous value, as the new $Q_{\text {leakage }}=0.003[\mathrm{l} / \mathrm{min}]$ based on Eq.1. In addition, a new adapted O-ring was added having an internal diameter of $23.34[\mathrm{~mm}]$ and torus diameter equal to $1.78[\mathrm{~mm}]$.

Finally, to overcome the pistons misalignment effects, a new solution is proposed. A new part called "piston support guide-way" denoted (F) on Fig.15, is added. This allows us to ensure an improved guidance of the hydraulic piston rod and thus prevents its misalignment by the radial force of the cables as shown in Fig. 14. An exploded view of the new mechanism with a reduced number of parts (10 including the active foot mechanism and its actuator) is given in Fig. 15. The new parts were manufactured and assembled as shown in Fig. 17. A CAD image for the new ankle mechanism with HYDROiD robot is shown in Fig.16.

\section{Experimental Results}

A setup dedicated to the analysis of the performances of the new enhanced version of the hybrid hydraulic ankle was developed. Fig. 17 shows an aluminum structure built to hold the fully parallel part and serial one of the ankle mechanism as well as the hydraulic actuator of the toe. This setup is supplied by an external HPU and can be loaded with several payloads. New joint sensors based on contact-less hall effect are 

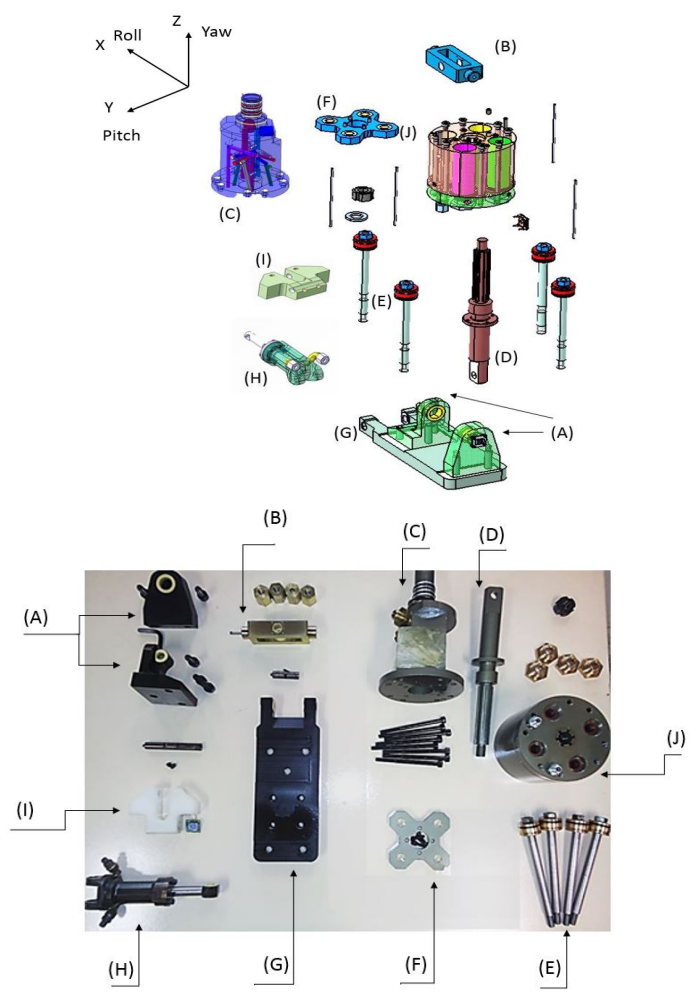

Fig. 15 Exploded CAD view (a) and photo of the enhanced ankle mechanism (b) with its main parts: (A) Foot supports, (B) Tibia axe support, (C)Upper tibia , (D) Tibia axe, (E) Pistons, (F) Piston guide-way, (G) Foot , (H) Toe piston, (I) Toe, (J) Tibia stator

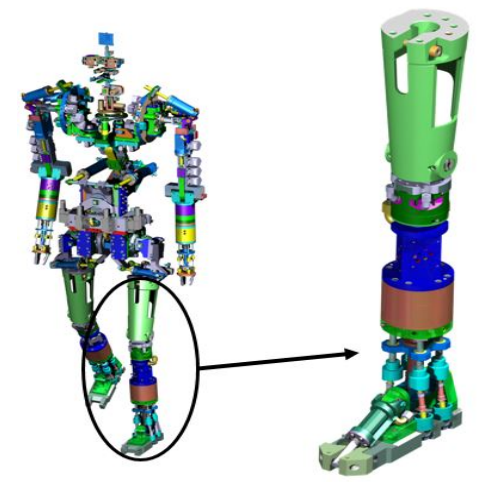

Fig. 16 HYDROiD humanoid robot including the new hybrid ankle mechanism

used for the new ankle mechanism. These sensors allow accurate measurement of the joint positions and allow driving the servo valves through control loop using a master 
control unit.

Our ultimate goal is to be able to reproduce, with the proposed solution, the performances of a human being who has the same weight of HYDROiD humanoid robot in terms of required torque and speed. A complete demonstration of the fully parallel degrees of freedom (ie. sagittal and frontal) as well as the toes movement are implemented. Fig. 18 is a snapshot extracted from video that shows that the required ranges of motions are successfully achieved. The upper and lower limits of the range of motion for both sagittal and frontal joints are $-15^{\circ}$ to $45^{\circ}$ and $-20^{\circ}$ to $20^{\circ}$ respectively. Such limits exceeds the previous limits of the first version of the ankle which were $-15^{\circ}$ to $30^{\circ}$ and $-15^{\circ}$ to $15^{\circ}$ for sagittal and frontal motions respectively.

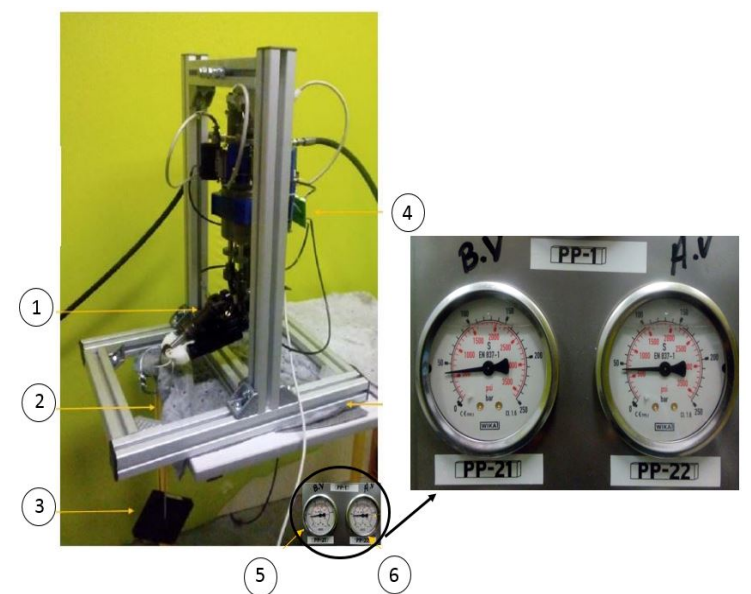

Fig. 17 A setup based on the enhanced version of HYDROïD hybrid ankle mechanism. Its main parts are (1)Toe piston, (2)Steel wire holding the weight, (3)Weight carried by the mechanism, (4)Electronic circuits and (5),(6)Pressure Gauges for the pressurized oil entering and exiting the cylinder

Position based control of motion of the frontal and sagittal joints is detailed in the following subsection. Moreover, a comparative study between the first and the enhanced versions of the torque that the ankle mechanism is able to produce, is also illustrated. This is given in subsection5.2, demonstrates the effectiveness of the new proposed solution.

\subsection{Experimental joint responses}

Experimental validation of the mechanism was carried for the frontal $\left(\theta_{x}\right)$ and the sagittal $\left(\theta_{y}\right)$ rotations. At first, flexion/extension motion $\theta_{y}$ was tested using two different inputs namely step and sinusoidal functions. For the step case, the response for an input of an amplitude almost equals to $23^{\circ}$ is shown in Fig.19, where the settling time is $0.09[s]$ and an overshoot of $0.796^{\circ}$ is observed. For a sinusoidal function 

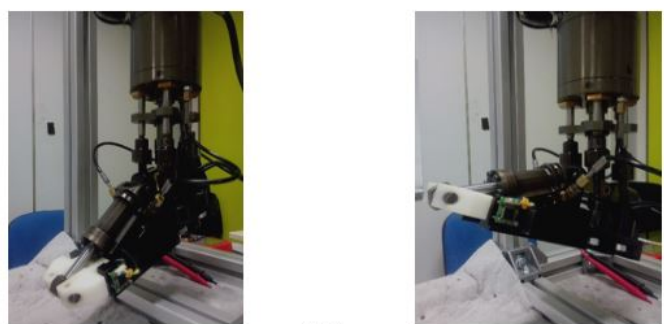

(A)
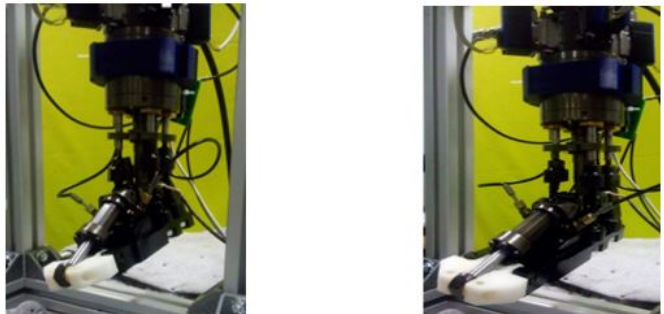

(B)

Fig. 18 The sagittal rotation (A) and the frontal rotation (B) for the HYDROiD ankle

input, the response for a sinusoidal of an amplitude almost equals to $6^{\circ}$ is given on Fig.20. In this case, the overshoot is found to be equal to $0^{\circ}$.

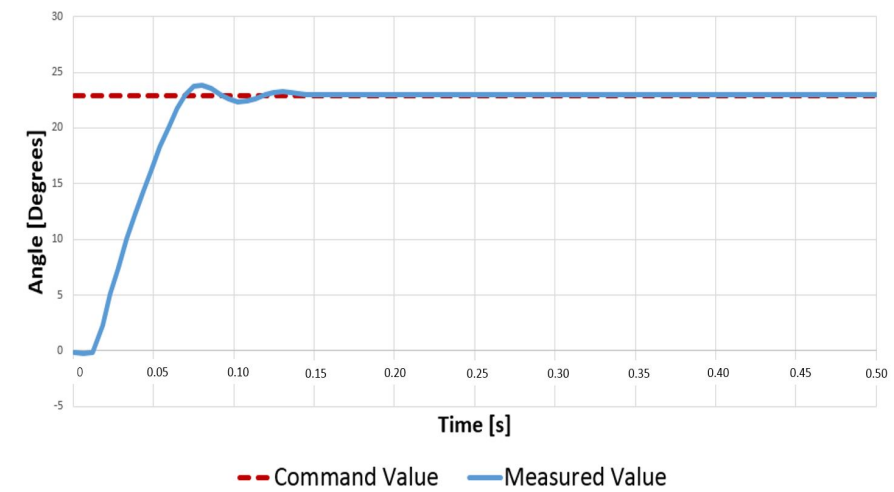

Fig. 19 Sagittal rotation response for a step input equal to $23^{\circ}$

Experimental validation of $\theta_{x}$ is shown in Fig.21. There is an overshoot almost equal to $0^{\circ}$. These results show clearly the experimental validity of the system response of the new modified mechanism. 


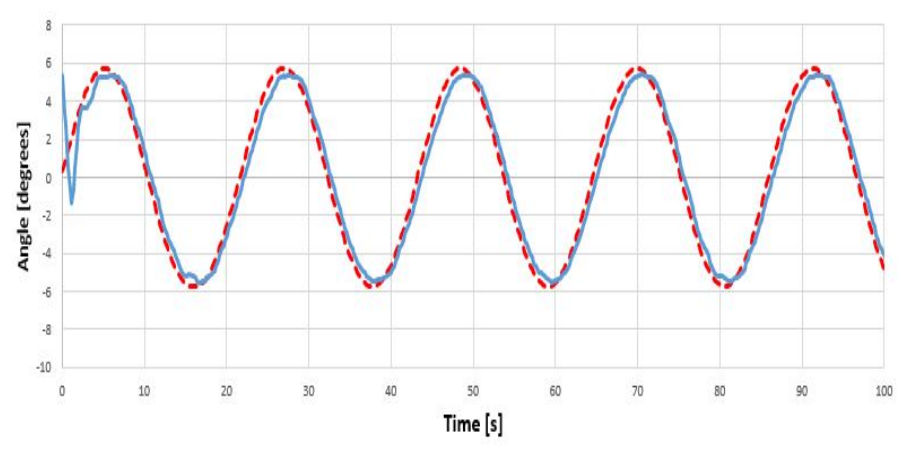

---Reference Values in degrees $\quad$ Measured Values in degrees

Fig. 20 Sagittal rotation response for sinusoidal input with an amplitude of $5.8^{\circ}$ and a frequency of $0.2[\mathrm{rad} / \mathrm{s}]$

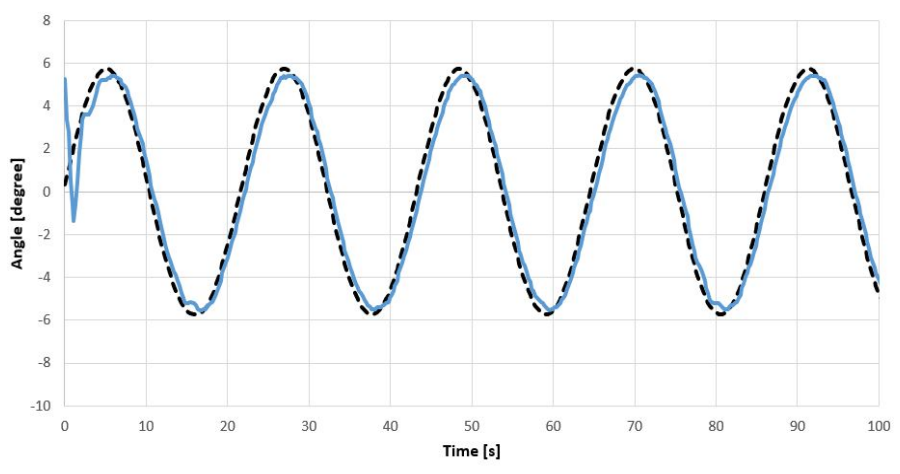

-- Reference Values in degrees —Measured Values in degrees

Fig. 21 Frontal rotation response for sinusoidal input with an amplitude of $5.8^{\circ}$ and a frequency of $0.2[\mathrm{rad} / \mathrm{s}]$

\subsection{Experimental required torque responses}

The ankle mechanism was tested to ensure that it will be able to achieve the walking gait. Since this mechanism is part of the underdeveloped HYDROï, walking is a premium objective to be achieved. Therefore, an average torque of 216 [N.m] is required for HYDROïD at its sagittal joint during the walking cycle [24]. Experimental study is conducted using the setup shown in Fig.17. The carried out tests include adding known blocks of masses held by a steel wire at the last point of the foot of the mechanism. The resultant static torque at the joint level is calculated analytically based on the gravity effects of the payload which include the mass of the foot $m_{\text {foot }}$ in addition to the known mass $m_{\text {load }}$. Knowing the distance between the point of fixation of the wire and the center of rotation of the foot denoted as $x_{\text {rotation }}$, the torque exerted by the ankle mechanism, denoted $T_{\text {sagittal }}$ can be easily calculated. By assuming that the foot center of mass is at a given distance from the center of ro- 
tation noticed $\lambda \times x_{\text {rotation }}$ with $(\lambda<1)$, thus the torque can be expressed as follows: $T_{\text {sagittal }}=F_{\text {load }} \times x_{\text {rotation }}$ and $F_{\text {load }}=\left(m_{\text {load }}+\lambda m_{\text {foot }}\right) \times g$.

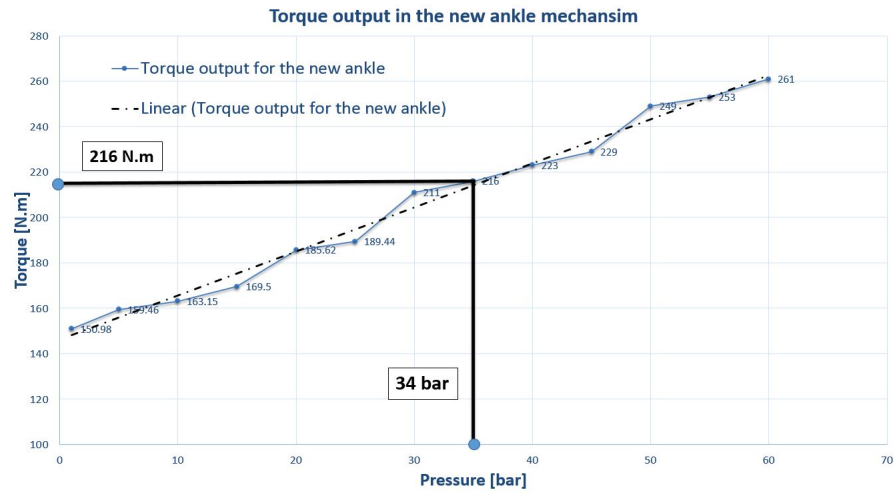

Fig. 22 Required torque comparison and related required pressure for both ankle versions

The HPU pressure required to achieve the needed torque for the old ankle of HYDROiD is almost 160 [bar] [24], while for the new ankle mechanism, a value of 34 [bar] (almost quarter of the initial one) is sufficient. The later value demonstrates the improvement of the performance of the enhanced version. While part of this improvement is due to increased diameters of the pistons, reducing friction forces plays a key role and consequently demonstrating the validity of the approach presented in this paper. Moreover, for a given HPU pressure, the new version of the ankle mechanism can produce larger torques allowing HYDROiD humanoid robot to deal with a wide range of external perturbations.

\section{Acknowledgment}

This work was mainly funded by BIA- TURNKEY TEST SYSTEMS Company and the French National Agency for Research (ANR).

\section{Conclusion and Future work}

In this paper, an enhanced hydraulic hybrid ankle mechanism was proposed. This mechanism showed a significant change to the properties of the system as a whole, mainly in producing larger torque at the joint levels, decreasing internal leakage and reducing friction effects. A virtual model for the hydraulic ankle mechanism was developed using ADAMS software in order to carry out a friction sensitivity analysis. A new modified orientation of the piston axes as well as a material selection have been proposed. Moreover, the introduction of "piston guide part" tackles the piston 
axes misalignment issue leading to more efficient solution. The new mechanism was manufactured, assembled and validated experimentally showing the increase of the efficiency of the new proposed hydraulic ankle.

For further work, the first step will be the evaluation of the dynamic performances of the ankle as part of one complete leg. Then, dynamic walking achievement of HYDROïD humanoid robot will be addressed.

\section{Nomenclature}

$\theta \quad$ Angle of rotation [degree]

$c_{f} \quad$ Coefficient of viscous friction $[\mathrm{N} . \mathrm{s} / \mathrm{mm}]$

$\mu_{f} \quad$ Coefficient of static friction $[-]$

$\beta \quad$ Bulk modulus of hydraulic oil $[\mathrm{GPa}]$

$Q_{\text {leakage }}$ Internal leakage flow rate $[l / \mathrm{min}]$

$v_{f} \quad$ Velocity of the piston during the transition phase $[\mathrm{mm} / \mathrm{s}]$

$v_{p_{i}} \quad$ Velocity of the piston $i[\mathrm{~mm} / \mathrm{s}]$

$S_{p} \quad$ cross-sectional area of the hydraulic piston $\left[\mathrm{m}^{2}\right]$

$F_{s} \quad$ Friction force of static friction $[N]$

$F_{f_{i}} \quad$ Total friction force which operates against the direction of piston $i$ movements. $[N]$

$\tau_{y}^{\max } \quad$ Maximum required torque in the sagittal plane $[N . m]$

$\alpha \quad$ Angle of piston misalignment with respect to cylinder center line [degrees]

$\tau_{x}^{\max } \quad$ Maximum required torque in the frontal plane $[N . m]$

$\tau_{y} \quad$ Flexion/Extenstion torque $[N . m]$

$\tau_{x} \quad$ Eversion/Inversion torque $[N . m]$

$\alpha \quad$ Rotating angle between the two hydraulic pistons couples around the roll axis [degree]

$X_{d} \quad$ Desired angles of rotation for the ankle [degree]

$X_{m} \quad$ Measured angles of rotation for the ankle [degree]

$L(i) \quad$ The stroke of the hydraulic piston $[\mathrm{mm}]$

$S C(i) \quad$ The outer diameter of the hydraulic pistons $[\mathrm{mm}]$

$E(i) \quad$ Piston connecting point with cables $[-]$

$A N(i) \quad$ Air pressure outlet point from the piston $[-]$

$\Delta P \quad$ Difference in pressure across the servo valve $[$ bar $]$

$F_{\text {load }} \quad$ Weight of the load applied on the foot $[N]$

$m_{\text {load }} \quad$ Mass of the load applied on the foot $[\mathrm{kg}]$

$m_{\text {foot }} \quad$ Mass of the foot $[\mathrm{kg}]$

$x_{\text {rotation }}$ Distance between the frontal end point of the foot with the point of rotation of the foot $[m]$

$T_{\text {rotation }}$ Rotation torque of the foot in the frontal plane $[N . m]$

$g \quad$ Gravitational constant $\left[\mathrm{m} / \mathrm{s}^{2}\right]$

$\theta_{x} \quad$ Angle of rotation in the frontal plane [degree]

$\theta_{y} \quad$ Angle of rotation in the sagittal plane [degree $]$ 
$\theta_{z} \quad$ Angle of rotation in the vertical plane [degree]

$\theta_{\text {toe }} \quad$ Angle of rotation of the toe of the ankle [degree]

$d \quad$ Piston diameter in $[m]$

I Servo valve current $[A]$

$K \quad$ Static flow gain constant $\left[\frac{\mathrm{m}^{3} / \mathrm{s}}{A}\right]$

$T \quad$ Time constant of first order equation $[s]$

$x_{v} \quad$ Displacement of the servo valve spool $[\mathrm{mm}]$

$\rho \quad$ Density of the hydraulic oil $\left[\mathrm{Kg} / \mathrm{m}^{3}\right]$

$c \quad$ Radial clearance $[m]$

$v \quad$ Kinematic viscosity $\left[\mathrm{m}^{2} / \mathrm{s}\right]$

\section{References}

1. S. Alfayad, F. Ouezdou, F. Namoun, Humanoid robot implementing a ball and socket joint, US Paten 12/991,443 (Jun 23, 2011).

2. H. Takanobu, E. Guglielmelli, H. Tabayashi, S. Narita, A. Takanishi, and P. Dario, Remote interaction between human and humanoid robot, J. Intell. Robot. Syst., p. V-2, 1998.

3. D. Katic and M. Vukobratovic, Survey of Intelligent Control Techniques for Humanoid Robots, J. Intell. Robot. Syst., pp. 117141, 2003.

4. Kazuo Hirai, Masato Hirose, Yuji Haikawa, and Toru Takenaka. The Development of Honda Humanoid Robot. In International Conference on Robotics and Automation, volume 2, pages 1321:1326, 1998.

5. David Gouaillier, Vincent Hugel, Pierre Blazevic, Chris Kilner, Jerome Monceaux, Pascal Lafourcade, Brice Marnier, Julien Serre, and Bruno Maisonnier. Mechatronic design of NAO humanoid. 2009 IEEE International Conference on Robotics and Automation, pages 769:774, 2009. ISSN 1050:4729.

6. H. Ulbrich, T. Buschmann, and S. Lohmeier, Design and realization of humanoid robots at AM-TUM, Proc. XII Intl. Symp. , no. Diname, 2007.

7. Yu Ogura, Hiroyuki Aikawa, Kazushi, Shimomura, Hideki Kondo, and Akitoshi Morishima, Hun-ok Lim Atsuo Takanishi, Development of a New Humanoid Robot WABIAN-2, Proc. of IEEE ICRA 2006, pp 76- 81, Orlando, Florida

8. S. H. Hyon, T. Yoneda, and D. Suewaka, Lightweight hydraulic leg to explore agile legged locomotion, IEEE Int. Conf. Intell. Robot. Syst., pp. 46554660, 2013.

9. Gordon Cheng, Sang Ho Hyon, Jun Morimoto, Aleš Ude, Glenn Colvin, Wayco Scroggin, and Stephen C. Jacobsen. CB: A humanoid research platform for exploring NeuroScience. Proceedings of the 2006 6th IEEE-RAS International Conference on Humanoid Robots, HUMANOIDS, 21(10), 182:187, 2006.

10. Darrin C. Bentivegna, Christopher G. Atkeson, and Jung Yup Kim. Compliant control of a hydraulic humanoid joint. Proceedings of the 2007 7th IEEERAS International Conference on Humanoid Robots, HUMANOIDS 2007, pages 483:489, 2008.

11. Claudio Semini, Nikos G. Tsagarakis, Emanuele Guglielmino, and Darwin G. Caldwell. Design and experimental evaluation of the hydraulically actuated prototype leg of the HyQ robot. In International Conference on Intelligent Robots and Systems - IROS, pages 3640:3645, 2010.

12. Scott Kuindersma, Robin Deits, Maurice Fallon Andr, Hongkai Dai, Frank Permenter, Koolen Pat, and Marion Russ. Optimization-based locomotion planning, estimation, and control design for the atlas humanoid robot. 2014.

13. S. Alfayad, F. B. Ouezdou, F. Namoun, O. Bruneau, and P. Hnaff, Three DOF hybrid mechanism for humanoid robotic application: Modeling, design and realization, 2009 IEEE/RSJ Int. Conf. Intell. Robot. Syst. IROS 2009, pp. 49554961, 2009.

14. S. H. Masaru Ogata, Study on Ankle Mechanisms for Walking Robots, J. Robot. Mechatronics, vol. Vol.16 No., no. 1, pp. 32013206, 2004.

15. C. E. Syrseloudis and I. Z. Emiris, A Parallel Robot for Ankle Rehabilitation-Evaluation and its Design. 8th IEEE International Conference on BioInformatics and BioEngineering. Pages: 1 - 6, DOI: 10.1109/BIBE.2008.4696826. 2008

16. F. Aggogeri, N. Pellegrini, and R. Adamini, Functional Design in Rehabilitation : Modular Mechanisms for Ankle Complex, vol. 2016, 2016. 
17. S. H. Member, D. Suewaka, Y. Torii, N. Oku, and H. Ishida, Design and Experimental Evaluation of a Fast Torque-Controlled Hydraulic Humanoid Robot. IEEE/ASME Transactions on Mechatronics. pp. 18. DOI: 10.1109/TMECH.2016.2628870. 2016

18. A. Omer, R. Ghorbani, K. Hashimoto, H. Lim, and A. Takanishi, A Novel Design for Adjustable Stiffness Artificial Tendon for the Ankle Joint of a Bipedal Robot : Modeling and Simulation, pp. 122, 2015.

19. K. Narioka, T. Homma, and K. Hosoda, Humanlike ankle-foot complex for a biped robot,. 12th IEEE-RAS International Conference on Humanoid Robots (Humanoids 2012). Pages: 15 - 20, DOI: 10.1109/HUMANOIDS.2012.6651493pp. 38. 2012

20. F. B. Ouezdou, S. Alfayad, and B. Almasri. Comparison of several kinds of feet for humanoid robot. Proceedings of 2005 5th IEEE-RAS International Conference on Humanoid Robots, 2005, 123:128, 2005.

21. A. David, J. R. L. A. Cnrs, J. R. L. A. Cnrs, J. R. L. A. Cnrs, and J. R. L. A. Cnrs, Study of an External Passive Shock-absorbing Mechanism for Walking Robots. Humanoids 2008 - 8th IEEE-RAS International Conference on Humanoid Robots. Pages: 435 - 440, DOI: 10.1109/ICHR.2008.4755991. 2008

22. Yousheng Yang; Semini, C.; Tsagarakis, N.G.; Guglielmino, E.; Caldwell, D.G., ’Leg mechanisms for hydraulically actuated robots," in Intelligent Robots and Systems, 2009. IROS 2009. IEEE/RSJ International Conference on , vol., no., pp.4669:4675, 10-15 Oct. 2009.

23. E. M. Ficanha, M. Rastgaar, and K. R. Kaufman, A two-axis cable-driven ankle-foot mechanism, Robot. Biomimetics, vol. 1, no. 1, p. 17, 2014.

24. S. Alfayad, F.B. Ouezdou, and Faycal Namoun. New 3-DOFs Hybrid Mechanism for Ankle and Wrist of Humanoid Robot: Modeling, Simulation, and Experiments. Journal of Mechanical Design, 133:021005, 2011. ISSN 10500472.

25. S. Alfayad, F. B. Ouezdou, F. Namoun, and G. Gheng, High performance Integrated Electro-Hydraulic Actuator for robotics. Part II: Theoretical modelling, simulation, control \& comparison with real measurements, Sensors Actuators, A Phys., vol. 169, pp. 124132, 2011.

26. Sumeet S. Aphale. Designing Orthogonal Gough Stewart Platforms with Robust Fault Tolerance. PhD thesis, Laramie, WY, USA, 2005. AAI3188203.

27. M. G. Rabie, Fluid Power Engineering. 2009, p. 420.

28. P.J. Gamez-Montero, E. Salazar, R. Castilla, J. Freire, M. Khamashta, and E. Codina. Misalignment effects on the load capacity of a hydraulic cylinder. International Journal of Mechanical Sciences, 51(2), $105: 113,2009$

29. F. Merritt, H. C. Systems, and J. Wiley, Electro-Hydraulic Servo Valve Construction, Models and Use, Control, 1967.

30. J. Faisandier. Mcanismes hydrauliques et pneumatiques. Collection : Technique et ingnierie, srie mcanique. Paris, 8 edition, 1999.

31. Xuan Bo Tran, Nur Hafizah, and Hideki Yanada. Modeling of dynamic friction behaviors of hydraulic cylinders. Mechatronics, 22, 65:75, 2012.

32. A.U Ellman, T.S. Koivula, and M.J. Vilenius. Hydraulic cylinder seal friction - comparison of two seal designs. 15th International Conference on Fluid Sealing, 1997. 